\title{
On the Hilbert symbol in cyclotomic fields
}

\author{
by \\ Charles Helou (Media, PA)
}

1. Introduction. Let $l$ be a prime number $\geq 5, \zeta$ a primitive $l$ th root of unity in an algebraic closure of the field $\mathbb{Q}_{l}$ of $l$-adic numbers, $K=\mathbb{Q}(\zeta)$, $\mathcal{O}_{K}=\mathbb{Z}[\zeta], \lambda=1-\zeta, \widehat{K}=\mathbb{Q}_{l}(\zeta)$, the $\lambda$-adic completion of $K$, and $\mathcal{O}_{\widehat{K}}=$ $\mathbb{Z}_{l}[\zeta]$, where $\mathbb{Z}_{l}$ is the ring of $l$-adic integers. The group of units of a ring $\mathcal{O}$ is denoted by $\mathcal{O}^{*}$. For $\alpha, \beta \in \widehat{K}^{*}$, we write

$$
(\alpha, \beta)_{\lambda}=\zeta^{[\alpha, \beta]} \text { with }[\alpha, \beta] \text { in } \mathbb{F}_{l}=\mathbb{Z} / l \mathbb{Z}
$$

for the Hilbert symbol as defined in [3], inverse of the one in [2]. Namely,

$$
(\alpha, \beta)_{\lambda}=\frac{\psi(\beta)\left(\alpha^{1 / l}\right)}{\alpha^{1 / l}},
$$

where $\psi: \widehat{K}^{*} \rightarrow \operatorname{Gal}\left(\widehat{K}\left(\alpha^{1 / l}\right) \mid \widehat{K}\right)$ is the local Artin map associated with the extension $\widehat{K}\left(\alpha^{1 / l}\right) \mid \widehat{K}$. This bilinear, skew-symmetric symbol defines an orthogonality relation in $\widehat{K}^{*}$ by the condition $[\alpha, \beta]=0$. Let $C$ be the group of cyclotomic units of $K$, i.e. the subgroup of $\mathcal{O}_{K}^{*}$ generated by the special units

$$
u_{k}=\frac{1-\zeta^{k}}{1-\zeta}=\frac{\sigma_{k}(\lambda)}{\lambda}
$$

where $\sigma_{k}$ is the element of the Galois group of $\widehat{K} \mid \mathbb{Q}_{l}$ defined by $\sigma_{k}(\zeta)=$ $\zeta^{k}(k \in \mathbb{Z} \backslash l \mathbb{Z})$. Fix an element $a \in \mathbb{Z} \backslash\{0\}$ and, for $n \in \mathbb{N} \backslash\{0\}$, let $\alpha_{n}=a^{n}-\zeta^{n}$. In 1989, G. Terjanian ([7]) conjectured that (TC) If $a \in \mathbb{Z} \backslash l \mathbb{Z}$ and $\alpha_{1}=a-\zeta$ is orthogonal to $C$, then $a \equiv \pm 1(\bmod l)$.

He showed that (TC) is true for the regular primes $l$ and for those for which $2^{l-1} \not \equiv 1\left(\bmod l^{2}\right)$ or the Bernoulli number $B_{l-3} \not \equiv 0(\bmod l)$. More recently, B. Anglès $([1])$ showed that Eichler's condition, $i(l)<\sqrt{l}-2$, for the index of irregularity $i(l)$ of the prime $l([8])$, implies that (TC) is true for $l$.

2000 Mathematics Subject Classification: 11R18, 11S31. 
In this paper, we study the following weak form of the conjecture:

(WTC) If $a \in \mathbb{Z} \backslash l \mathbb{Z}$ and, for all $n \in \mathbb{N} \backslash l \mathbb{N}, \alpha_{n}=a^{n}-\zeta^{n}$ is orthogonal to $C$, then $a \equiv \pm 1(\bmod l)$.

We can also state (TC) and (WTC) in an equivalent form using the group $\mu_{l-1}$ of $(l-1)$ th roots of unity in $\mathbb{Z}_{l}^{*}$. Indeed, for any $a \in \mathbb{Z} \backslash l \mathbb{Z}$, there is an $\omega \in \mu_{l-1}$ such that $\omega \equiv a(\bmod l)$, namely $\omega=\lim _{n \rightarrow \infty} a^{l^{n}}([4])$. Therefore $\omega \equiv a^{l}\left(\bmod l^{2}\right)$ and, by the properties of the Hilbert symbol, $\left[\omega^{n}-\zeta^{n}, u\right]=\left[a^{l n}-\zeta^{n}, u\right]$ for all $u \in \mathbb{Z}_{l}^{*}$ and $n \in \mathbb{N} \backslash l \mathbb{N}$. Thus, $\omega^{n}-\zeta^{n}$ is orthogonal to $C$ if and only if $a^{l n}-\zeta^{n}$ is. Moreover, if $a^{n}-\zeta^{n}$ is orthogonal to $C(n \in \mathbb{N} \backslash l \mathbb{N})$, then $a^{l-1} \equiv 1\left(\bmod l^{2}\right)([7])$ and $\omega^{n}-\zeta^{n} \equiv a^{l n}-\zeta^{n} \equiv a^{n}-\zeta^{n}$ $\left(\bmod l^{2}\right)$, so that $\omega^{n}-\zeta^{n}=\sigma_{n}\left(\omega^{n}-\zeta\right)$ is orthogonal to $C$, i.e. $\omega^{n}-\zeta$ is orthogonal to $C$. Also, since $\omega^{l^{k}}=\omega(k \in \mathbb{N})$ and $\omega^{-1}=\omega^{l-2}$, we see that, for any $m \in \mathbb{Z} \backslash\{0\}, \omega^{m}-\zeta=\omega^{n}-\zeta$ for some $n \in \mathbb{N} \backslash l \mathbb{N}$; as to $m=0$, the element $\lambda$ is anyway orthogonal to $C$. Therefore the assumption in (TC) (resp. in (WTC)) entails the orthogonality of $\omega-\zeta$ (resp. of $\omega^{m}-\zeta$, for all $m \in \mathbb{Z})$ to $C$. On the other hand, $a \equiv \pm 1(\bmod l)$ if and only if $\omega \equiv \pm 1$ $(\bmod l)$, which, by a simple induction argument, amounts to $\omega=\omega^{l^{n}} \equiv \pm 1$ $\left(\bmod l^{n+1}\right)$ for all $n \geq 1$, i.e. to $\omega= \pm 1$. It follows that (TC) and (WTC) are respectively equivalent to

$$
\text { If } \omega \in \mu_{l-1} \text { and } \omega-\zeta \text { is orthogonal to } C \text {, then } \omega^{2}=1 \text {. }
$$

(WTC) If $\omega \in \mu_{l-1}$ and, for all $n \in \mathbb{Z}, \omega^{n}-\zeta$ is orthogonal to $C$, then $\omega^{2}=1$.

In fact, the assumption in (WTC) is equivalent to: $\omega^{n}-\zeta$ is orthogonal to $C$ for $1 \leq n \leq f-1$, where $f$ is the order of $\omega$.

We first derive some properties of the Hilbert symbol and some explicit expressions obtained via the Artin-Hasse reciprocity law, that we need in what follows. We then establish orthogonality relations between some classes of elements $\omega^{m}-\zeta$ and $\sigma_{k}\left(\omega^{n}-\zeta\right)$ and deduce the validity of (WTC) under certain conditions. Thus if (WTC) fails for $l$ then there exists a divisor $f \geq$ 11 of $l-1$ such that for any divisor $d$ of $f, d^{l-1} \equiv 1\left(\bmod l^{2}\right)$. Furthermore, (WTC) is true for every prime $l$ of one of the following forms: $l=2^{n}+1$; or $l=2^{n}-1$; or $l=2^{h_{0}} p^{h}+1$ with $h_{0} \leq 3, p$ prime and $h \geq 1$; or $l=2^{h_{0}} p_{1}^{h_{1}} p_{2}^{h_{2}}+1$ with $h_{0} \leq 3, p_{1}, p_{2}$ primes and $h_{1}, h_{2} \geq 1$ such that $h_{i} q\left(p_{i}\right) \not \equiv 1(\bmod l)(i=1,2)$; or $l=2^{h_{0}} p_{1}^{h_{1}} \ldots p_{m}^{h_{m}}$, where the $p_{i}$ are primes and the $h_{i} \geq 1$ are such that $\sum_{i=1}^{m} h_{i} q\left(p_{i}\right) \not \equiv 1(\bmod l)$ or $h_{0} \leq 3$ and $p_{i}^{l-1} \not \equiv 1\left(\bmod l^{2}\right)$ for $1 \leq i \leq m$. Here $q(x)=\left(x^{l-1}-1\right) / l$ is the Fermat quotient for $x \in \mathbb{Z}_{l}^{*}$.

2. Properties of the Hilbert symbol. The Hilbert symbol has the following fundamental properties (see [2, Ch. 12, §1]). 
Lemma 1. For any $x, y, z \in \widehat{K}^{*}$, we have:

(1) $[x y, z]=[x, z]+[y, z] ;\left[x^{n}, y\right]=\left[x, y^{n}\right]=n[x, y](n \in \mathbb{Z})$; $[y, x]=-[x, y] ;[x, \pm x]=0$.

(2) If $x \neq 1$ then $[x, 1-x]=0$. If $x \neq-1$ then $[x, 1+x]=0$.

(3) If $x \neq-y$ then $[x, y]=[x, x+y]+[x+y, y]$. If $x \neq y$ then $[x, y]=[x, x-y]+[x-y, y]$.

Lemma 2. (1) For $x, y \in \widehat{K}^{*}$ and $k \in \mathbb{Z} \backslash l \mathbb{Z},\left[\sigma_{k}(x), \sigma_{k}(y)\right]=k[x, y]$.

(2) If $F$ is a proper subfield of $\widehat{K}$ and $x, y \in F^{*}$ then $[x, y]=0$.

(3) The group $C$ of cyclotomic units is invariant under the action of $\operatorname{Gal}\left(\widehat{K} \mid \mathbb{Q}_{l}\right)$, i.e. $\sigma_{k}(C)=C$ for any $k \in \mathbb{Z} \backslash l \mathbb{Z}$.

We denote by $v_{\lambda}$ the normalized $\lambda$-adic valuation of $\widehat{K}$.

Lemma 3. (1) If $\alpha \in \mathcal{O}_{\widehat{K}}^{*}$ satisfies $\alpha^{l-1} \equiv 1\left(\bmod \lambda^{l+1}\right)$, then $\alpha$ is orthogonal to $\widehat{K}^{*}$. In particular, any $\omega \in \mu_{l-1}$ is orthogonal to $\widehat{K}^{*}$.

(2) If $x_{1}, x_{2}, y_{1}, y_{2} \in \widehat{K}^{*}$ are such that $v_{\lambda}\left(x_{1}\right)=v_{\lambda}\left(x_{2}\right)=h, v_{\lambda}\left(y_{1}\right)=$ $v_{\lambda}\left(y_{2}\right)=k, v_{\lambda}\left(x_{1}-x_{2}\right) \geq l+h+1$ and $v_{\lambda}\left(y_{1}-y_{2}\right) \geq l+k+1$, then $\left[x_{1}, y_{1}\right]=\left[x_{2}, y_{2}\right]$.

Proof. (1) By an application of Hensel's lemma, a unit of $\widehat{K}$ which is congruent to an $l$ th power $\left(\bmod \lambda^{l+1}\right)$ is an $l$ th power in $\widehat{K}([2$, Ch. 12 , Lemma 4]). In particular, if $\alpha^{l-1} \equiv 1\left(\bmod \lambda^{l+1}\right)$ then $\alpha^{l-1}=\gamma^{l}$ for some $\gamma \in \mathcal{O}_{\widehat{K}}^{*}$ and $[\alpha, y]=-\left[\alpha^{l-1}, y\right]=-l[\gamma, y]=0$.

(2) We have $x_{i}=\lambda^{h} \alpha_{i}$ and $y_{i}=\lambda^{k} \beta_{i}$, with $\alpha_{i}, \beta_{i} \in \mathcal{O}_{\widehat{K}}^{*}(i=1,2)$. Moreover, $v_{\lambda}\left(\alpha_{1}-\alpha_{2}\right)=v_{\lambda}\left(x_{1}-x_{2}\right)-h \geq l+1$, i.e. $\alpha_{1} \alpha_{2}^{-1} \equiv 1\left(\bmod \lambda^{l+1}\right)$; and similarly $\beta_{1} \beta_{2}^{-1} \equiv 1\left(\bmod \lambda^{l+1}\right)$. Then, by $(1), x_{1} x_{2}^{-1}=\alpha_{1} \alpha_{2}^{-1}$ and $y_{1} y_{2}^{-1}=\beta_{1} \beta_{2}^{-1}$ are orthogonal to $\widehat{K}^{*}$. In particular, $\left[x_{1} x_{2}^{-1}, y_{1}\right]=0$ and $\left[x_{2}, y_{1} y_{2}^{-1}\right]=0$, i.e. $\left[x_{1}, y_{1}\right]=\left[x_{2}, y_{1}\right]$ and $\left[x_{2}, y_{1}\right]=\left[x_{2}, y_{2}\right]$. Hence the result.

Lemma 4. (1) If $\alpha, \beta \in \mathcal{O}_{\widehat{K}}^{*}$ are such that $\alpha^{l-1} \equiv 1\left(\bmod \lambda^{i}\right)$ and $\beta^{l-1} \equiv$ $1\left(\bmod \lambda^{j}\right)$, with $i, j \in \mathbb{N}$, and $i+j \geq l+1$, then $[\alpha, \beta]=0$.

(2) If $\alpha \in \mathcal{O}_{\widehat{K}}^{*}$ satisfies $\alpha^{l-1} \equiv 1\left(\bmod \lambda^{l}\right)$, then $\alpha$ is orthogonal to $\mathcal{O}_{\widehat{K}}^{*}$.

(3) If $\alpha_{1}, \alpha_{2}, \beta_{1}, \beta_{2} \in \mathcal{O}_{\widehat{K}}^{*}$ are such that $\alpha_{1}^{l-1} \equiv \alpha_{2}^{l-1}\left(\bmod \lambda^{l}\right)$ and $\beta_{1}^{l-1} \equiv \beta_{2}^{l-1}\left(\bmod \lambda^{l}\right)$, then $\left[\alpha_{1}, \beta_{1}\right]=\left[\alpha_{2}, \beta_{2}\right]$.

Proof. (1) If $i=0$ or $j=0$, the result follows from Lemma 3(1). Moreover, since $[\alpha, \beta]=\left[\alpha^{l-1}, \beta^{l-1}\right]$, we may then assume that $\alpha \equiv 1\left(\bmod \lambda^{i}\right)$ and $\beta \equiv 1\left(\bmod \lambda^{j}\right)$, with $i, j \geq 1$ and $i+j \geq l+1$. The proof uses the multiplicative basis $\eta_{i}=1-\lambda^{i}(i \geq 1)$ of the principal units (i.e. those $\equiv 1$ $(\bmod \lambda))$ of $\widehat{K}$. 
Note first that the property holds for the $\eta_{i}$ 's. Indeed, from the relation $\eta_{i+j}=\eta_{j}+\lambda^{j} \eta_{i}$, we deduce, via Lemma 1 , that $\left[\eta_{i}, \eta_{j}\right]=\left[\eta_{i}, \eta_{i+j}\right]+$ $\left[\eta_{i+j}, \eta_{j}\right]+j\left[\lambda, \eta_{i+j}\right]$; and each of the last three symbols is zero, by Lemma $3(1)$.

Then the property is extended to all the principal units, using a descending induction on $i+j$ and the fact that we can write $\alpha=\eta_{i}^{m} \alpha^{\prime}$ and $\beta=\eta_{j}^{n} \beta^{\prime}$, with $m, n \in \mathbb{Z}$ and $\alpha^{\prime}, \beta^{\prime} \in \mathcal{O}_{\widehat{K}}$ such that $\alpha^{\prime} \equiv 1\left(\bmod \lambda^{i+1}\right)$ and $\beta^{\prime} \equiv 1$ $\left(\bmod \lambda^{j+1}\right)$. Indeed, since by the binomial formula $\eta_{i}^{k} \equiv 1-k \lambda^{i}\left(\bmod \lambda^{i+1}\right)$ (for $k \in \mathbb{Z}$ ) and since $(1-\alpha) / \lambda^{i}$ is in $\mathcal{O}_{\widehat{K}}$ and is thus $\equiv m(\bmod \lambda)$ for some $m \in \mathbb{Z}$, it follows that we may take $\alpha^{\prime}=\alpha \eta_{i}^{-m}$, as it is $\equiv 1\left(\bmod \lambda^{i+1}\right)$; similarly for $\beta^{\prime}$. Therefore $[\alpha, \beta]=m n\left[\eta_{i}, \eta_{j}\right]+m\left[\eta_{i}, \beta^{\prime}\right]+n\left[\alpha^{\prime}, \eta_{j}\right]+\left[\alpha^{\prime}, \beta^{\prime}\right]$, where the last three symbols are zero by the induction assumption and the one before them is zero by the property for the $\eta_{i}$ 's. Hence the result in general.

(2) For $\beta \in \mathcal{O}_{\widehat{K}}^{*}$, we have $\beta^{l-1} \equiv 1(\bmod \lambda)$, and since $\alpha^{l-1} \equiv 1\left(\bmod \lambda^{l}\right)$, (1) shows that $[\alpha, \beta]=0$.

(3) We have $\left(\alpha_{1} \alpha_{2}^{-1}\right)^{l-1} \equiv 1\left(\bmod \lambda^{l}\right)$ and thus, by $(2),\left[\alpha_{1} \alpha_{2}^{-1}, \beta_{1}\right]=0$, i.e. $\left[\alpha_{1}, \beta_{1}\right]=\left[\alpha_{2}, \beta_{1}\right]$. Similarly, $\left[\alpha_{2}, \beta_{1} \beta_{2}^{-1}\right]=0$, i.e. $\left[\alpha_{2}, \beta_{1}\right]=\left[\alpha_{2}, \beta_{2}\right]$. Hence the result.

LEMMA 5. (1) If $x, y \in K^{*}$ are of the form $x=\lambda^{h} u$ and $y=\lambda^{k} v$, where $u, v \in \mathcal{O}_{K}^{*}$ are global units and $h, k \in \mathbb{Z}$, then $[x, y]=0$.

(2) The $\mathbb{Q}$-conjugates of $\zeta$, of $\lambda$ and of the elements of $C$ are pairwise orthogonal.

(3) If $\omega \in \mu_{l-1}$ has order $f$, then, for any $n \in \mathbb{Z}$ such that $f \mid 2 n, \omega^{n}-\zeta$ is orthogonal to its $\mathbb{Q}_{l}$-conjugates, to those of $\lambda$ and to $C$.

Proof. (1) By the product formula for the Hilbert symbols over all the primes (finite or infinite) $\mathfrak{p}$ of $K$ ([2, Ch. 12, Theorem 13]), for $x, y \in K^{*}$, $\prod_{\mathfrak{p}}(x, y)_{\mathfrak{p}}=1$. Moreover, from the properties of the local Artin maps, if $\mathfrak{p}$ is finite, $\mathfrak{p} \nmid l$ and the $\mathfrak{p}$-adic valuations $v_{\mathfrak{p}}(x)=v_{\mathfrak{p}}(y)=0$, then $(x, y)_{\mathfrak{p}}=1$. Also, for the infinite primes $\mathfrak{p}$, the completion of $K$ at $\mathfrak{p}$ is $\simeq \mathbb{C}$ and thus $(x, y)_{\mathfrak{p}}=1$. Therefore, for $x, y$ as in the statement, $(x, y)_{\mathfrak{p}}=1$ for all $\mathfrak{p} \neq(\lambda)$. Thus, the product formula reduces to $(x, y)_{\lambda}=1$, i.e. $[x, y]=0$.

(2) follows immediately from (1).

(3) Since $f \mid 2 n$, we see that $\omega^{2 n}=1$, i.e. $\omega^{n}= \pm 1$. Hence $\omega^{n}-\zeta=$ $\pm 1-\zeta=\lambda$ or $=-(1+\zeta)=-\sigma_{2}(\lambda) / \lambda=-u_{2}$, which lies in $C$. Hence the result, by (2).

Lemma 6. (1) If $\alpha, \beta \in \mathcal{O}_{\widehat{K}}^{*}$ and $a_{0}, a_{1}, b \in \mathbb{Z}_{l}$ are such that $\alpha \equiv a_{0}+a_{1} \lambda$ $\left(\bmod \lambda^{2}\right)$ and $\beta \equiv b\left(\bmod \lambda^{l}\right)$, then $[\alpha, \beta]=\left(a_{1} / a_{0}\right) q(b)$. 
(2) For $u, v, x, y \in \mathbb{Z}_{l}$ such that $l \nmid(u+v)(x+y)$, we have

$$
[u+v \zeta, x+y \zeta]=\frac{(u y-v x)^{l}-(u+v) y^{l}+v^{l}(x+y)}{l(u+v)(x+y)} .
$$

(3) If $\alpha \in \mathcal{O}_{\widehat{K}}^{*}$, then $[\alpha, \zeta]=(N(\alpha)-1) / l$, where $N$ is the norm in $\widehat{K} \mid \mathbb{Q}_{l}$.

(4) If $a \in \mathbb{Z}_{l}^{*}$, then $[a, \lambda]=\frac{1}{2} q(a)$.

Proof. (1) By Lemma 4, $[\alpha, \beta]=[\alpha, b]$. Then, by [6, Corollary 1 to Theorem 2 and the Remark after Corollary 2],

$$
[\alpha, b]=\frac{a_{1}}{a_{0}} \cdot \frac{b^{l-1}-1}{l} .
$$

Hence the result.

(2) This results from [6, Theorem 3 and its Corollary 2] by the same calculation that gave the expression for

$$
\left(\frac{x+y \zeta}{u+v \zeta}\right)_{l}\left(\frac{u+v \zeta}{x+y \zeta}\right)_{l}^{-1}=[u+v \zeta, x+y \zeta],
$$

which, in view of the note following that theorem, is valid for $[u+v \zeta, x+y \zeta]$ when $u, v, x, y \in \mathbb{Z}_{l}$.

(3) This results from [6, Theorem 2 and the Remark after its Corollary 2], since $\zeta=1-\lambda$ and $\log \zeta=0$.

(4) By [2, Ch. 12, Th. 10], whose third part is missing a factor $1 / \lambda$ of $\zeta \log \alpha$, and in which the symbol is the opposite of the one in [3], used here, we have

$$
[a, \lambda]=-\frac{1}{l} \operatorname{Tr}\left(\frac{\zeta}{\lambda} \log a\right)
$$

where $\operatorname{Tr}$ is the trace map in $\widehat{K} \mid \mathbb{Q}_{l}$. Since $a \in \mathbb{Z}_{l}^{*}$, we find that $a^{l-1} \equiv 1$ $(\bmod l)$ and

$$
\log a=\frac{1}{l-1} \log \left(a^{l-1}\right) \equiv \frac{1}{l-1}\left(a^{l-1}-1\right) \equiv-\left(a^{l-1}-1\right)\left(\bmod l^{2}\right) .
$$

Hence

$$
\frac{\zeta}{\lambda} \log a \equiv-\frac{\zeta}{\lambda}\left(a^{l-1}-1\right)\left(\bmod l^{2} \mathfrak{D}^{-1}\right),
$$

where $\mathfrak{D}=\left(\lambda^{l-2}\right)$ is the different ideal of $\widehat{K} \mid \mathbb{Q}_{l}$, and therefore

$$
\operatorname{Tr}\left(\frac{\zeta}{\lambda} \log a\right) \equiv-\left(a^{l-1}-1\right) \operatorname{Tr}\left(\frac{\zeta}{\lambda}\right)\left(\bmod l^{2}\right)
$$

(cf. [5, p. 150]). Moreover, $\operatorname{Tr}(\zeta / \lambda)=\operatorname{Tr}(1 / \lambda-1)=\operatorname{Tr}(1 / \lambda)-(l-1)$ and, 
by $[5$, p. 173], $\operatorname{Tr}(1 / \lambda)=(l-1) / 2$. Thus

$$
\operatorname{Tr}\left(\frac{\zeta}{\lambda} \log a\right) \equiv \frac{l-1}{2}\left(a^{l-1}-1\right) \equiv-\frac{1}{2}\left(a^{l-1}-1\right)\left(\bmod l^{2}\right) .
$$

The result follows by substitution into the expression of $[a, \lambda]$.

Lemma 7. Let $a \in \mathbb{Z}_{l}^{*}$.

(1) If $a^{2} \not \equiv 1(\bmod l)$ then

$$
\left[a-\zeta, a^{2}-\zeta^{2}\right]=\frac{2 a}{a^{2}-1}(q(2)+q(a)) .
$$

(2) If $a^{3} \not \equiv 1(\bmod l)$ then

$$
\left[a-\zeta, a^{3}-\zeta^{3}\right]=\frac{3 a(a+1)}{2\left(a^{3}-1\right)}(q(3)+2 q(a)),
$$

where, for any $x \in \mathbb{Z}_{l}^{*}, q(x)=\left(x^{l-1}-1\right) / l$.

Proof. We use the notation $\alpha_{n}=a^{n}-\zeta^{n}$.

(1) Since $\alpha_{2}=(a+\zeta) \alpha_{1}$, we see that $\left[\alpha_{1}, \alpha_{2}\right]=[a-\zeta, a+\zeta]$. By Lemma 6(2),

$$
[a-\zeta, a+\zeta]=\frac{(2 a)^{l}-2 a}{l\left(a^{2}-1\right)}=\frac{2 a}{a^{2}-1} q(2 a) .
$$

The result follows by noting that $q(2 a) \equiv q(2)+q(a)(\bmod l)([5$, Lemma 2$])$.

(2) Since $\alpha_{3}=\left(a^{2}+a \zeta+\zeta^{2}\right) \alpha_{1}$, we find that $\left[\alpha_{1}, \alpha_{3}\right]=\frac{1}{2}\left[\alpha_{1}^{2}, a^{2}+a \zeta+\zeta^{2}\right]$. By Lemma 1, $\left[\alpha_{1}^{2}, a^{2}+a \zeta+\zeta^{2}\right]=\left[\alpha_{1}^{2}, 3 a \zeta\right]+\left[3 a \zeta, \alpha_{3} / \alpha_{1}\right]$. Hence $\left[\alpha_{1}, \alpha_{3}\right]=$ $\frac{3}{2}\left[\alpha_{1}, 3 a \zeta\right]-\frac{1}{2}\left[\alpha_{3}, 3 a \zeta\right]$. For $n=1$ or 3 , we have $\left[\alpha_{n}, 3 a \zeta\right]=\left[\alpha_{n}, 3 a\right]+\left[\alpha_{n}, \zeta\right]$, and since $\alpha_{n}=a^{n}-(1-\lambda)^{n} \equiv a^{n}-1+n \lambda\left(\bmod \lambda^{2}\right)$, Lemma 6 shows that

$$
\left[\alpha_{n}, 3 a\right]=\frac{n}{a^{n}-1} q(3 a) \quad \text { and } \quad\left[\alpha_{n}, \zeta\right]=\frac{N\left(\alpha_{n}\right)-1}{l} .
$$

Moreover, since $N\left(\alpha_{n}\right)=\left(a^{n l}-1\right) /\left(a^{n}-1\right)$, it follows that

$$
\frac{N\left(\alpha_{n}\right)-1}{l}=\frac{a^{n l}-a^{n}}{l\left(a^{n}-1\right)}=\frac{a^{n}}{a^{n}-1} q\left(a^{n}\right) .
$$

Therefore,

$$
\left[\alpha_{n}, 3 a \zeta\right]=\frac{n}{a^{n}-1}\left(q(3)+\left(a^{n}+1\right) q(a)\right)
$$

(using the additivity of $q(x)(\bmod l)[5$, Lemma 2$])$. The result now follows by substitution.

Lemma 8. Let $\omega \in \mu_{l-1}$.

(1) For any $n \in \mathbb{Z}$ and $k \in \mathbb{Z} \backslash l \mathbb{Z},\left[\omega^{n} \pm \zeta^{k}, \zeta\right]=0$.

(2) For any $j, m, n \in \mathbb{Z}$ and $k \in \mathbb{Z} \backslash l \mathbb{Z},\left[\omega^{-m}-\zeta^{-j}, \omega^{n}-\zeta^{k}\right]=\left[\omega^{m}-\zeta^{j}\right.$, $\left.\omega^{n}-\zeta^{k}\right]$. 
(3) For any $j, k, m, n \in \mathbb{Z}$ such that $l \nmid j$,

$\left[\omega^{m}-\zeta^{j}, \omega^{n}-\zeta^{k}\right]=\left[\omega^{m}-\zeta^{j}, \omega^{m-n}-\zeta^{j-k}\right]+\left[\omega^{m-n}-\zeta^{j-k}, \omega^{n}-\zeta^{k}\right]$.

(4) For any $j, k, m, n \in \mathbb{Z}$ such that $l \nmid j+k$, $\left[\omega^{m}-\zeta^{j}, \omega^{n}-\zeta^{k}\right]=\left[\omega^{m}-\zeta^{j}, \omega^{m+n}-\zeta^{j+k}\right]+\left[\omega^{m+n}-\zeta^{j+k}, \omega^{n}-\zeta^{k}\right]$.

Proof. (1) By Lemma 1,

$$
\left[\omega^{n} \pm \zeta^{k}, \zeta\right]=\frac{1}{k}\left[\omega^{n} \pm \zeta^{k}, \zeta^{k}\right]=\frac{1}{k}\left(\left[\omega^{n} \pm \zeta^{k}, \omega^{n}\right]+\left[\omega^{n}, \zeta^{k}\right]\right)
$$

The last two symbols are zero since, by Lemma $3, \omega$ is orthogonal to $\widehat{K}^{*}$.

(2) We have $\omega^{-m}-\zeta^{-j}=-\omega^{-m} \zeta^{-j}\left(\omega^{m}-\zeta^{j}\right)$. Therefore $\left[\omega^{-m}-\zeta^{-j}, \omega^{n}-\zeta^{k}\right]=\left[\omega^{m}-\zeta^{j}, \omega^{n}-\zeta^{k}\right]-m\left[\omega, \omega^{n}-\zeta^{k}\right]-j\left[\zeta, \omega^{n}-\zeta^{k}\right]$. The latter symbol is zero by (1) above, and the one before it is zero since $\omega$ is orthogonal to $\widehat{K}^{*}$. Hence the result.

(3) We have $\omega^{n}-\zeta^{k}=\omega^{n-m}\left(\omega^{m}-\omega^{m-n} \zeta^{k}\right)$. Since $\omega$ is orthogonal to $\widehat{K}^{*}$, it follows that $\left[\omega^{m}-\zeta^{j}, \omega^{n}-\zeta^{k}\right]=\left[\omega^{m}-\zeta^{j}, \omega^{m}-\omega^{m-n} \zeta^{k}\right]$. By Lemma 1 , we have

$$
\begin{aligned}
{\left[\omega^{m}-\zeta^{j}, \omega^{m}-\omega^{m-n} \zeta^{k}\right]=} & {\left[\omega^{m}-\zeta^{j}, \omega^{m-n} \zeta^{k}-\zeta^{j}\right] } \\
& +\left[\omega^{m-n} \zeta^{k}-\zeta^{j}, \omega^{m}-\omega^{m-n} \zeta^{k}\right] .
\end{aligned}
$$

Since $\omega^{m-n} \zeta^{k}-\zeta^{j}=\zeta^{k}\left(\omega^{m-n}-\zeta^{j-k}\right)$ and, by (1) above, as $l \nmid j, \zeta$ is orthogonal to $\omega^{m}-\zeta^{j}$, we find that $\left[\omega^{m}-\zeta^{j}, \omega^{m-n} \zeta^{k}-\zeta^{j}\right]=\left[\omega^{m}-\zeta^{j}, \omega^{m-n}-\right.$ $\left.\zeta^{j-k}\right]$. Also, and since $\omega$ is orthogonal to $\widehat{K}^{*}$, we have

$$
\begin{aligned}
{\left[\omega^{m-n} \zeta^{k}-\zeta^{j}, \omega^{m}-\omega^{m-n} \zeta^{k}\right] } & =\left[\zeta^{k}\left(\omega^{m-n}-\zeta^{j-k}\right), \omega^{n}-\zeta^{k}\right] \\
& =\left[\zeta^{k}, \omega^{n}-\zeta^{k}\right]+\left[\omega^{m-n}-\zeta^{j-k}, \omega^{n}-\zeta^{k}\right]
\end{aligned}
$$

and $\left[\zeta^{k}, \omega^{n}-\zeta^{k}\right]=\left[\zeta^{k}, \omega^{n}\right]+\left[\omega^{n}, \omega^{n}-\zeta^{k}\right]=0$. Therefore

$$
\left[\omega^{m-n} \zeta^{k}-\zeta^{j}, \omega^{m}-\omega^{m-n} \zeta^{k}\right]=\left[\omega^{m-n}-\zeta^{j-k}, \omega^{n}-\zeta^{k}\right]
$$

and the result follows.

(4) It follows from (3) that

$$
\left[\omega^{m+n}-\zeta^{j+k}, \omega^{n}-\zeta^{k}\right]=\left[\omega^{m+n}-\zeta^{j+k}, \omega^{m}-\zeta^{j}\right]+\left[\omega^{m}-\zeta^{j}, \omega^{n}-\zeta^{k}\right] .
$$

Hence the result.

3. Orthogonality conditions. In what follows, $\omega$ will denote an element of $\mu_{l-1}$ of order $f$.

Proposition 1. Assume that, for some $n \in \mathbb{Z}, \omega^{n}-\zeta$ is orthogonal to $C$. Then

(1) $\omega^{n}-\zeta$ is orthogonal to its $\mathbb{Q}_{l}$-conjugates and to those of $\lambda$. 
(2) If $\omega^{2 n} \neq 1$, then $\left(\omega^{n}-1\right)^{l-1} \equiv\left(\omega^{n}+1\right)^{l-1} \equiv 1\left(\bmod l^{2}\right)$, and thus $\omega^{n} \pm 1$ are orthogonal to $\widehat{K}^{*}$.

Proof. By the same arguments as in the proof of [5, Proposition 6(b)], applied to $\omega^{n}$ instead of $a$, since $\omega^{n}-\zeta$ is orthogonal to $C$, it is orthogonal to its $\mathbb{Q}_{l}$-conjugates and to those of $\lambda$. In particular, $\left[\omega^{n}-\zeta, \lambda\right]=0$. But, by Lemma 1 ,

$$
\left[\omega^{n}-\zeta, \lambda\right]=\left[\omega^{n}-1+\lambda, \lambda\right]=\left[\omega^{n}-1+\lambda, \omega^{n}-1\right]+\left[\omega^{n}-1, \lambda\right] .
$$

And, by Lemma 6 , since $\omega^{n} \neq 1$, we have

$$
\left[\omega^{n}-1+\lambda, \omega^{n}-1\right]=\frac{1}{\omega^{n}-1} q\left(\omega^{n}-1\right) \quad \text { and } \quad\left[\omega^{n}-1, \lambda\right]=\frac{1}{2} q\left(\omega^{n}-1\right) .
$$

Thus

$$
\left[\omega^{n}-\zeta, \lambda\right]=\frac{\omega^{n}+1}{2\left(\omega^{n}-1\right)} q\left(\omega^{n}-1\right)=0,
$$

and since $\omega^{n} \neq-1$, this implies that $q\left(\omega^{n}-1\right)=0$ in $\mathbb{F}_{l}$, i.e. $\left(\omega^{n}-1\right)^{l-1} \equiv 1$ $\left(\bmod l^{2}\right)$. On the other hand, $\omega^{n}-\zeta$ is also orthogonal to the cyclotomic unit $u_{2}=1+\zeta$. Therefore, similarly using Lemmas 1 and 6 , we get

$$
\begin{aligned}
{\left[\omega^{n}-\zeta, 1+\zeta\right] } & =\left[\omega^{n}-1+\lambda, \omega^{n}+1\right]+\left[\omega^{n}+1,2-\lambda\right] \\
& =\frac{1}{\omega^{n}-1} q\left(\omega^{n}+1\right)+\frac{1}{2} q\left(\omega^{n}+1\right) \\
& =\frac{\omega^{n}+1}{2\left(\omega^{n}-1\right)} q\left(\omega^{n}+1\right)=0,
\end{aligned}
$$

which means that $\left(\omega^{n}+1\right)^{l-1} \equiv 1\left(\bmod l^{2}\right)$. We conclude, by Lemma 3 , that $\omega^{n}-1$ and $\omega^{n}+1$ are orthogonal to $\widehat{K}^{*}$.

Proposition 2. (1) If $\omega^{n}-\zeta$ is orthogonal to $C$ for $1 \leq n \leq f-1$, then $\omega^{n}-\zeta$ is orthogonal to $C$ for all $n \in \mathbb{Z}$.

(2) If $\left[\omega^{m}-\zeta, \sigma_{k}\left(\omega^{n}-\zeta\right)\right]=0$ for $1 \leq m, n \leq f-1,1 \leq k \leq l-1$, then $\omega^{n}-\zeta$ is orthogonal to $C$ and $\left[\omega^{m}-\zeta, \sigma_{k}\left(\omega^{n}-\zeta\right)\right]=0$ for all $m, n \in \mathbb{Z}$, $k \in \mathbb{Z} \backslash l \mathbb{Z}$.

Proof. (1) For any $n \in \mathbb{Z}$, if $f \nmid n$, then there is some $1 \leq r \leq f-1$ such that $n \equiv r(\bmod f)$. Then $\omega^{n}-\zeta=\omega^{r}-\zeta$ is, by assumption, orthogonal to $C$. While if $f \mid n$, then $\omega^{n}-\zeta=\lambda$ is, by Lemma 5 , orthogonal to $C$.

(2) For $1 \leq n \leq f-1$ and $2 \leq k \leq l-1$, we have

$$
\omega^{n}-\zeta-\sigma_{k}\left(\omega^{n}-\zeta\right)=-\zeta \sigma_{k-1}(\lambda)
$$

and thus, by Lemma 1 and the assumption, $\left[\omega^{n}-\zeta, \sigma_{k}\left(\omega^{n}-\zeta\right)\right]=\left[\omega^{n}-\zeta,-\zeta \sigma_{k-1}(\lambda)\right]+\left[-\zeta \sigma_{k-1}(\lambda), \sigma_{k}\left(\omega^{n}-\zeta\right)\right]=0$. Moreover, by Lemma $8,\left[\omega^{n}-\zeta, \zeta\right]=\left[\zeta, \sigma_{k}\left(\omega^{n}-\zeta\right)\right]=0$. Hence $\left[\omega^{n}-\zeta\right.$, $\left.\sigma_{k-1}(\lambda)\right]=\left[\sigma_{k}\left(\omega^{n}-\zeta\right), \sigma_{k-1}(\lambda)\right]$. Letting $h$ denote the inverse of $(k-1)$ 
$(\bmod l)$ lying between 1 and $l-1$, we deduce, using Lemma 2 , that

$$
\left[\sigma_{h}\left(\omega^{n}-\zeta\right), \lambda\right]=\left[\sigma_{h+1}\left(\omega^{n}-\zeta\right), \lambda\right] \quad \text { for } 1 \leq h \leq l-2 .
$$

Thus $\left[\sigma_{k}\left(\omega^{n}-\zeta\right), \lambda\right]=\left[\omega^{n}-\zeta, \lambda\right]$ for $1 \leq k \leq l-1$; and therefore

$$
\left[N\left(\omega^{n}-\zeta\right), \lambda\right]=\sum_{k=1}^{l-1}\left[\sigma_{k}\left(\omega^{n}-\zeta\right), \lambda\right]=(l-1)\left[\omega^{n}-\zeta, \lambda\right],
$$

where $N$ is the norm map in $\widehat{K} \mid \mathbb{Q}_{l}$. Moreover,

$$
N\left(\omega^{n}-\zeta\right)=\frac{\omega^{n l}-1}{\omega^{n}-1}=1 .
$$

Hence $\left[\sigma_{k}\left(\omega^{n}-\zeta\right), \lambda\right]=-\left[N\left(\omega^{n}-\zeta\right), \lambda\right]=0$ for $1 \leq k \leq l-1$. Therefore, by Lemma $2, \omega^{n}-\zeta$ is orthogonal to the $\mathbb{Q}$-conjugates of $\lambda$, and thus to $C$. This holds for $1 \leq n \leq f-1$, and thus, by (1), for all $n \in \mathbb{Z}$.

Now, for any $m, n \in \mathbb{Z}$, if $f \nmid m$ and $f \nmid n$, then $m \equiv r(\bmod f)$ and $n \equiv s(\bmod f)$, for some $1 \leq r, s \leq f-1$, so that $\omega^{m}-\zeta=\omega^{r}-\zeta$ and $\sigma_{k}\left(\omega^{n}-\zeta\right)=\sigma_{k}\left(\omega^{s}-\zeta\right)$ are, by assumption, orthogonal (for $1 \leq k \leq l-1$ ). While if $f \mid n$, then the $\mathbb{Q}_{l}$-conjugates of $\omega^{n}-\zeta=\lambda$ are, by Proposition 1 , orthogonal to $\omega^{m}-\zeta$ and, by Lemma 5 , to those of $\lambda$. A similar conclusion is reached if $f \mid m$. Hence $\left[\omega^{m}-\zeta, \sigma_{k}\left(\omega^{n}-\zeta\right)\right]=0$ in all cases.

In what follows we make the following assumption:

$$
\text { For all } n \in \mathbb{Z}, \omega^{n}-\zeta \text { is orthogonal to } C \text {. }
$$

Proposition 3. Under assumption (A), we have:

(1) For any $n \in \mathbb{Z}$ such that $f \nmid 2 n$, we have $\left(\omega^{n}-1\right)^{l-1} \equiv\left(\omega^{n}+1\right)^{l-1} \equiv 1$ $\left(\bmod l^{2}\right)$, and thus $\omega^{n} \pm 1$ are orthogonal to $\widehat{K}^{*}$.

(2) For any $m, n \in \mathbb{Z}$ with $2 m \not \equiv 2 n(\bmod f)$, we have $\left(\omega^{m}-\omega^{n}\right)^{l-1} \equiv$ $\left(\omega^{m}+\omega^{n}\right)^{l-1} \equiv 1\left(\bmod l^{2}\right)$, and thus $\omega^{m} \pm \omega^{n}$ are orthogonal to $\widehat{K}^{*}$.

(3) For any $n \in \mathbb{Z}$, the $\mathbb{Q}_{l}$-conjugates of the elements $\lambda$, $\omega^{n}-\zeta, \omega^{-n}-\zeta$ are pairwise orthogonal.

Proof. (1) This follows from Proposition 1.

(2) This follows from (1) since $\omega^{m} \pm \omega^{n}=\omega^{n}\left(\omega^{m-n} \pm 1\right)$ and $f \nmid 2(m-n)$.

(3) By Proposition 1 and Lemma 5, the $\mathbb{Q}_{l}$-conjugates of $\lambda$ and $\omega^{n}-\zeta$ (resp. of $\lambda$ and $\omega^{-n}-\zeta$ ) are pairwise orthogonal. Moreover, for $k \in \mathbb{Z} \backslash l \mathbb{Z}$, $\sigma_{k}\left(\omega^{-n}-\zeta\right)=-\omega^{-n} \zeta^{k} \sigma_{-k}\left(\omega^{n}-\zeta\right)$ is a product of elements which are orthogonal to $\omega^{n}-\zeta$ and its conjugates. Thus the conjugates of $\omega^{-n}-\zeta$ are orthogonal to those of $\omega^{n}-\zeta$.

Proposition 4. Under assumption (A), we have, for $m, n \in \mathbb{Z}$ and $k \in$ $\mathbb{Z} \backslash l \mathbb{Z}:$

(1) If $f \mid 2 m$ or $f \mid 2 n$, then $\left[\omega^{m}-\zeta, \sigma_{k}\left(\omega^{n}-\zeta\right)\right]=0$. 
(2) If $m \equiv \pm 2 n(\bmod f)$ or $n \equiv \pm 2 m(\bmod f)$, then

$$
\left[\omega^{m}-\zeta, \sigma_{k}\left(\omega^{n}-\zeta\right)\right]=0 .
$$

Proof. By the skew-symmetry of the symbol and Lemma 2, it is enough to only consider one of the two conditions in each case.

(1) Assume that $f \mid 2 n$. Then $\omega^{n}= \pm 1$ and $\omega^{n}-\zeta= \pm 1-\zeta=\lambda$ or $=-(1+\zeta)=-u_{2}$, which lies in $C$. Therefore, by assumption (A) and Proposition $1, \omega^{m}-\zeta$ is orthogonal to the $\mathbb{Q}_{l}$-conjugates of $\omega^{n}-\zeta$.

(2) Since, by Lemmas 8 and 2 ,

$$
\left[\omega^{-m}-\zeta, \omega^{n}-\zeta^{k}\right]=\left[\omega^{m}-\zeta^{-1}, \omega^{n}-\zeta^{k}\right]=-\left[\omega^{m}-\zeta, \omega^{n}-\zeta^{-k}\right],
$$

we may just assume $m \equiv 2 n(\bmod f)$ and, in view of $(1), f \nmid 2 n$. Then $\omega^{m}=\omega^{2 n}$ and $\omega^{m}-\zeta=\left(\omega^{n}-\zeta^{h}\right)\left(\omega^{n}+\zeta^{h}\right)$, where $h=(l+1) / 2$. Hence $\left[\omega^{m}-\zeta, \omega^{n}-\zeta^{k}\right]=\left[\omega^{n}-\zeta^{h}, \omega^{n}-\zeta^{k}\right]+\left[\omega^{n}+\zeta^{h}, \omega^{n}-\zeta^{k}\right]$. By Proposition 1, $\left[\omega^{n}-\zeta^{h}, \omega^{n}-\zeta^{k}\right]=0$. Thus, Lemma 1 yields

$$
\begin{aligned}
{\left[\omega^{m}-\zeta, \omega^{n}-\zeta^{k}\right] } & =\left[\omega^{n}+\zeta^{h}, \omega^{n}-\zeta^{k}\right] \\
& =\left[\omega^{n}+\zeta^{h}, \zeta^{k}\left(1+\zeta^{h-k}\right)\right]+\left[\zeta^{k}\left(1+\zeta^{h-k}\right), \omega^{n}-\zeta^{k}\right] .
\end{aligned}
$$

For $k \neq h, 1 \leq k \leq l-1$, the element $1+\zeta^{h-k}=u_{2(h-k)} / u_{h-k}$ is the quotient of two cyclotomic units, so that $\zeta^{k}\left(1+\zeta^{h-k}\right)$ lies in $C$. By assumption (A), $\omega^{n}-\zeta$ and $\omega^{2 n}-\zeta$ are orthogonal to $C$, and thus, by Lemma 2 , their $\mathbb{Q}_{l}$-conjugates are also orthogonal to $C$. Therefore $\omega^{n}-\zeta^{k}=\sigma_{k}\left(\omega^{n}-\zeta\right)$ and $\omega^{n}+\zeta^{h}=\left(\omega^{2 n}-\zeta\right) /\left(\sigma_{h}\left(\omega^{n}-\zeta\right)\right)$ are orthogonal to $\zeta^{k}\left(1+\zeta^{h-k}\right)$. It follows that $\left[\omega^{m}-\zeta, \omega^{n}-\zeta^{k}\right]=0$ for $1 \leq k \leq l-1, k \neq h$. Moreover, since $f \nmid n$, the norm $N\left(\omega^{n}-\zeta\right)$ is 1 and thus $\sum_{k=1}^{l-1}\left[\omega^{m}-\zeta, \omega^{n}-\zeta^{k}\right]=$ $\left[\omega^{m}-\zeta, N\left(\omega^{n}-\zeta\right)\right]=0$. It follows that also $\left[\omega^{m}-\zeta, \omega^{n}-\zeta^{h}\right]=0$. Hence $\left[\omega^{m}-\zeta, \sigma_{k}\left(\omega^{n}-\zeta\right)\right]=0$ for $1 \leq k \leq l-1$.

Proposition 5. Under assumption (A), we have, for $m, n \in \mathbb{Z}$ and $k \in$ $\mathbb{Z} \backslash l \mathbb{Z}$

$$
\left[\omega^{m}-\zeta^{k}, \omega^{n}-\zeta^{k}\right]=\left[\omega^{m}-\zeta^{k}, \omega^{n}-\zeta^{-k}\right]=0 .
$$

Proof. Since, by Lemma 8, $\left[\omega^{m}-\zeta^{k}, \omega^{n}-\zeta^{-k}\right]=\left[\omega^{m}-\zeta^{k}, \omega^{-n}-\zeta^{k}\right]$, it is enough to get $\left[\omega^{m}-\zeta^{k}, \omega^{n}-\zeta^{k}\right]=0$ for all $m, n, k(l \nmid k)$. If $2 m \equiv 2 n$ $(\bmod f)$, then either $\omega^{m}=\omega^{n}$ and the result is trivially true; or $\omega^{m}=-\omega^{n}$, in which case $\omega^{m}-\zeta^{k}=-\left(\omega^{2 n}-\zeta^{2 k}\right) /\left(\omega^{n}-\zeta^{k}\right)$, so that

$$
\left[\omega^{m}-\zeta^{k}, \omega^{n}-\zeta^{k}\right]=\left[\omega^{2 n}-\zeta^{2 k}, \omega^{n}-\zeta^{k}\right]=k\left[\sigma_{2}\left(\omega^{2 n}-\zeta\right), \omega^{n}-\zeta\right]=0,
$$

by Proposition 4 . Assume now that $2 m \not \equiv 2 n(\bmod f)$. Then, by Lemma 1 ,

$$
\left[\omega^{m}-\zeta^{k}, \omega^{n}-\zeta^{k}\right]=\left[\omega^{m}-\zeta^{k}, \omega^{m}-\omega^{n}\right]+\left[\omega^{m}-\omega^{n}, \omega^{n}-\zeta^{k}\right],
$$

and by Proposition $3, \omega^{m}-\omega^{n}$ is orthogonal to $\widehat{K}^{*}$. Hence $\left[\omega^{m}-\zeta^{k}, \omega^{n}-\zeta^{k}\right]$ $=0$. 
Proposition 6. Under assumption (A), for $m, n \in \mathbb{Z}$ and $k \in \mathbb{Z} \backslash l \mathbb{Z}$, if $2 m \equiv \pm 2 n(\bmod f)$, then $\left[\omega^{m}-\zeta, \sigma_{k}\left(\omega^{n}-\zeta\right)\right]=0$.

Proof. In view of Lemma 8 , we may just assume that $2 m \equiv 2 n(\bmod f)$, so that $\omega^{m}= \pm \omega^{n}$. If $\omega^{m}=\omega^{n}$, the result follows from Proposition 1. If $\omega^{m}=-\omega^{n}$, then $\omega^{m}-\zeta=-\left(\omega^{2 n}-\zeta^{2}\right) /\left(\omega^{n}-\zeta\right)$ and, by Proposition 1, $\omega^{n}-\zeta$ is orthogonal to its $\mathbb{Q}_{l}$-conjugates, so that $\left[\omega^{m}-\zeta, \sigma_{k}\left(\omega^{n}-\zeta\right)\right]=$ $\left[\omega^{2 n}-\zeta^{2}, \sigma_{k}\left(\omega^{n}-\zeta\right)\right]$. The latter symbol is, by Lemma 2 , equal to $2\left[\omega^{2 n}-\zeta, \sigma_{k(l+1) / 2}\left(\omega^{n}-\zeta\right)\right]$, which, by Proposition 4 , is equal to 0 . Hence the result.

\section{Conclusions}

Theorem 1. Let $\omega \in \mu_{l-1}$, of order $f$, satisfy the assumption (A) For all $n \in \mathbb{Z}, \omega^{n}-\zeta$ is orthogonal to $C$. If $\omega^{2} \neq 1$, i.e. if $f>2$, then:

(1) $2^{l-1} \equiv 1\left(\bmod l^{2}\right)$.

(2) For any divisor $d$ of $f$ in $\mathbb{N}, d^{l-1} \equiv 1\left(\bmod l^{2}\right)$.

Proof. (1) Since $\omega^{2} \neq 1$, we have $\omega^{2} \not \equiv 1(\bmod l)$. Indeed the congruence $\omega^{2} \equiv 1(\bmod l)$ implies, by induction, $\omega^{2}=\omega^{2 l^{n}} \equiv 1\left(\bmod l^{n+1}\right)$ for all $n \in \mathbb{N}$, which implies $\omega^{2}=1$. Therefore, by Lemma 7 , we have

$$
\left[\omega-\zeta, \omega^{2}-\zeta^{2}\right]=\frac{2 \omega}{\omega^{2}-1} q(2),
$$

since $q(\omega)=0$. On the other hand, by Proposition $4(2),\left[\omega-\zeta, \omega^{2}-\zeta^{2}\right]=0$. It follows that $q(2)=0$ in $\mathbb{F}_{l}$, i.e. $2^{l-1} \equiv 1\left(\bmod l^{2}\right)$.

(2) We may assume $d>1$. Let $e=f / d$ and $\gamma=\omega^{e}$. Then $\gamma$ is a primitive $d$ th root of unity in $\mathbb{Z}_{l}^{*}$. Hence $X^{d}-1=\prod_{j=0}^{d-1}\left(X-\gamma^{j}\right)$. Dividing by $X-1$, we get $\sum_{j=0}^{d-1} X^{j}=\prod_{j=1}^{d-1}\left(X-\gamma^{j}\right)$. Substituting $X=1$, we deduce that $d=\prod_{j=1}^{d-1}\left(1-\gamma^{j}\right)=\prod_{j=1}^{d-1}\left(1-\omega^{e j}\right)$. For $1 \leq j \leq d-1$, we have $1 \leq e j<e d=f$, so that $f \nmid e j$. If furthermore $f \nmid 2 e j$ then, by Proposition $3,\left(1-\omega^{e j}\right)^{l-1} \equiv 1\left(\bmod l^{2}\right)$. If however $f \mid 2 e j$ then, since $f \nmid e j$, we have $\omega^{e j}=-1$, so that $\left(1-\omega^{e j}\right)^{l-1}=2^{l-1} \equiv 1\left(\bmod l^{2}\right)$, by $(1)$ above. Thus, for all $1 \leq j \leq d-1$, we have $\left(1-\omega^{e j}\right)^{l-1} \equiv 1\left(\bmod l^{2}\right)$, and therefore $d^{l-1}=\prod_{j=1}^{d-1}\left(1-\omega^{e j}\right)^{l-1} \equiv 1\left(\bmod l^{2}\right)$.

COROLlary 1. Under assumption (A), we have:

(1) If $l$ is not of the form $2^{n}+1$ then there exists an odd prime factor $p$ of $l-1$ which does not divide $f$.

(2) If $l=2^{n} \pm 1$, for some positive integer $n$, then $f=1$ or 2 .

(3) If $l \geq 7$ then $f \leq(l-1) / 3$. 
Proof. (1) By assumption, $l-1$ has at least one odd prime factor. If $f$ is divisible by every odd prime factor $p$ of $l-1$, then $f>2$ and, by Theorem 1 , $p^{l-1} \equiv 1\left(\bmod l^{2}\right)$ for all such $p$, as well as for $p=2$. Therefore $l-1$, which is a product of powers of those primes, also satisfies $(l-1)^{l-1} \equiv 1\left(\bmod l^{2}\right)$. But, by the binomial formula, $(l-1)^{l-1} \equiv 1+l \not \equiv 1\left(\bmod l^{2}\right)$, a contradiction. Hence the result.

(2) If $f>2$ then, by Theorem $1,2^{l-1} \equiv 1\left(\bmod l^{2}\right)$. Since $l=2^{n} \pm 1$, it follows that $(l \mp 1)^{l-1}=2^{n(l-1)} \equiv 1\left(\bmod l^{2}\right)$. But, by the binomial formula, $(l \mp 1)^{l-1} \equiv 1 \pm l \not \equiv 1\left(\bmod l^{2}\right)$, a contradiction. Hence the result.

(3) If $l-1$ is not a power of 2 then, by (1), there is an odd prime factor $p$ of $l-1$ which does not divide $f$; hence $p f \mid l-1$ and thus $f \leq(l-1) / p \leq$ $(l-1) / 3$. If $l-1$ is a power of 2 then, by (2), $f \leq 2$ and, since $l \geq 7$, this gives $f \leq(l-1) / 3$.

ThEOREM 2. Let $\omega \in \mu_{l-1}$, of order $f$, satisfy the assumption

For all $n \in \mathbb{Z}, \omega^{n}-\zeta$ is orthogonal to $C$.

If $f \leq 10$ then, for all $m, n \in \mathbb{Z}$ and $k \in \mathbb{Z} \backslash l \mathbb{Z},\left[\omega^{m}-\zeta, \sigma_{k}\left(\omega^{n}-\zeta\right)\right]=0$.

Proof. For any $m, n \in \mathbb{Z}$, there exist integers $0 \leq r, s \leq f / 2$ such that $m \equiv \pm r(\bmod f)$ and $n \equiv \pm s(\bmod f)$. If one of the integers $2 r, 2 s, 2(r \pm s)$, $2 r \pm s, r \pm 2 s$ is divisible by $f$, then, by Propositions 4 and 6 , $\left[\omega^{m}-\zeta\right.$, $\left.\sigma_{k}\left(\omega^{n}-\zeta\right)\right]=0$. Taking into account the skew-symmetry of the symbol, we are reduced to considering the pairs $(r, s)$ such that $0 \leq r \leq s \leq f / 2$. Moreover, if $r=0$ or $s=f / 2$ then $f \mid 2 r$ or $f \mid 2 s$; if $r=s$ or $s=f / 2-r$ then $f \mid 2(r \pm s)$; if $s=2 r$ or $f-2 r$ then $f \mid 2 r \pm s$; if $s=(f-r) / 2$ then $f \mid r+2 s$. In all these cases, as explained above, we have $\left[\omega^{m}-\zeta, \sigma_{k}\left(\omega^{n}-\zeta\right)\right]=0$.

Thus, it only remains to consider the pairs $(r, s)$ such that $1 \leq r<$ $s<f / 2$ and $s \neq 2 r, f-2 r, f / 2-r,(f-r) / 2$. Let $E_{f}$ be the set of pairs $(r, s)$ of integers satisfying these conditions. It is easy to check that for $1 \leq f \leq 8, E_{f}=\emptyset$ and thus the result is established for these values of $f$; while $E_{9}=\{(1,3),(2,3),(3,4)\}$ and $E_{10}=\{(1,3)\}$.

Let $f=9$. Consider first the case $(r, s)=(1,3)$, i.e. $m \equiv \pm 1(\bmod f)$ and $n \equiv \pm 3(\bmod f)$. As, by Lemma $8(2)$ and Lemma $2,\left[\omega^{ \pm m}-\zeta, \sigma_{k}\left(\omega^{ \pm n}-\zeta\right)\right]=$ $\epsilon_{1}\left[\omega^{m}-\zeta, \sigma_{\epsilon_{2} k}\left(\omega^{n}-\zeta\right)\right]$ with $\epsilon_{1}, \epsilon_{2}= \pm 1$, we may assume that $m \equiv 1$ $(\bmod f)$ and $n \equiv 3(\bmod f)$. Since $l \nmid k$, there is a unique integer $h$ such that $1 \leq h \leq l-1$ and $h k \equiv 1(\bmod l)$. Then, for $0 \leq j \leq h-1$, we have $l \nmid(1-j k)$ and, by Lemma $8(3)$,

$$
\begin{aligned}
{\left[\omega^{m-j n}-\zeta^{1-j k}, \omega^{n}-\zeta^{k}\right]=} & {\left[\omega^{m-j n}-\zeta^{1-j k}, \omega^{m-(j+1) n}-\zeta^{1-(j+1) k}\right] } \\
& +\left[\omega^{m-(j+1) n}-\zeta^{1-(j+1) k}, \omega^{n}-\zeta^{k}\right] .
\end{aligned}
$$

Moreover, $m-j n \equiv 1-3 j(\bmod f), m-(j+1) n \equiv-2-3 j(\bmod f)$ and $-2-3 j \equiv-2(1-3 j)(\bmod f)$, so that, by Proposition 4 , for $0 \leq$ 
$j \leq h-2$, we have $\left[\omega^{m-j n}-\zeta^{1-j k}, \omega^{m-(j+1) n}-\zeta^{1-(j+1) k}\right]=0$ and thus $\left[\omega^{m-j n}-\zeta^{1-j k}, \omega^{n}-\zeta^{k}\right]=\left[\omega^{m-(j+1) n}-\zeta^{1-(j+1) k}, \omega^{n}-\zeta^{k}\right]$. It follows that $\left[\omega^{m}-\zeta, \omega^{n}-\zeta^{k}\right]=\left[\omega^{m-(h-1) n}-\zeta^{1-(h-1) k}, \omega^{n}-\zeta^{k}\right]$ and, by Lemma 8 , this is equal to $\left[\omega^{m-(h-1) n}-\zeta^{1-(h-1) k}, \omega^{m-h n}-\zeta^{1-h k}\right]+\left[\omega^{m-h n}-\zeta^{1-h k}, \omega^{n}-\zeta^{k}\right]$. Since $l \mid(1-h k)$ and $2(m-h n) \equiv 2(1-3 h) \not \equiv 0(\bmod f)$, Proposition 3 shows that $\omega^{m-h n}-\zeta^{1-h k}=\omega^{m-h n}-1$ is orthogonal to $\widehat{K}^{*}$. Hence $\left[\omega^{m}-\zeta\right.$, $\left.\omega^{n}-\zeta^{k}\right]=0$.

The remaining two cases $(r, s)=(2,3)$ or $(3,4)$ now follow from the previous ones. Indeed, we may, as before, assume that $m \equiv 2(\bmod f)$ and $n \equiv 3(\bmod f)(\operatorname{resp} . m \equiv 3(\bmod f)$ and $n \equiv 4(\bmod f))$. We may also assume that $k \not \equiv 1(\bmod l)$, otherwise we conclude by Proposition 5 . By Lemma 8,

$$
\left[\omega^{m}-\zeta, \omega^{n}-\zeta^{k}\right]=\left[\omega^{m}-\zeta, \omega^{m-n}-\zeta^{1-k}\right]+\left[\omega^{m-n}-\zeta^{1-k}, \omega^{n}-\zeta^{k}\right] .
$$

Since $m-n \equiv-1(\bmod f)$, from the case $(r, s)=(1,3)$ we deduce that $\left[\omega^{m-n}-\zeta^{1-k}, \omega^{n}-\zeta^{k}\right]=0$ (resp. $\left.\left[\omega^{m}-\zeta, \omega^{m-n}-\zeta^{1-k}\right]=0\right)$. Moreover, by Proposition 4 , i.e. by the case $r \equiv \pm 2 s(\bmod f)$, we get $\left[\omega^{m}-\zeta, \omega^{m-n}-\zeta^{1-k}\right]$ $=0\left(\right.$ resp. $\left.\left[\omega^{m-n}-\zeta^{1-k}, \omega^{n}-\zeta^{k}\right]=0\right)$. Hence $\left[\omega^{m}-\zeta, \omega^{n}-\zeta^{k}\right]=0$.

Let $f=10$ and consider the remaining case $(r, s)=(1,3)$. Then, as before, we may assume that $m \equiv 1(\bmod f), n \equiv 3(\bmod f)$ and $k \not \equiv 1$ $(\bmod l)$. By Lemma 8 again,

$$
\left[\omega^{m}-\zeta, \omega^{n}-\zeta^{k}\right]=\left[\omega^{m}-\zeta, \omega^{m-n}-\zeta^{1-k}\right]+\left[\omega^{m-n}-\zeta^{1-k}, \omega^{n}-\zeta^{k}\right] .
$$

Since $m-n \equiv-2(\bmod f)$, we have $m-n \equiv-2 m(\bmod f)$ and $2(m-n) \equiv 2 n$ $(\bmod f)$. Therefore, by Propositions 4 and $6,\left[\omega^{m}-\zeta, \omega^{m-n}-\zeta^{1-k}\right]=0$ and $\left[\omega^{m-n}-\zeta^{1-k}, \omega^{n}-\zeta^{k}\right]=0$. Hence $\left[\omega^{m}-\zeta, \omega^{n}-\zeta^{k}\right]=0$.

COROllary 2. Under assumption $(\mathrm{A})$, if $f \leq 10$ then $\omega^{2}=1$ (i.e. $f \leq 2$ ).

Proof. By Theorem 2, $\left[\omega^{m}-\zeta, \sigma_{k}\left(\omega^{n}-\zeta\right)\right]=0$ for all $m, n \in \mathbb{Z}$ and $k \in \mathbb{Z} \backslash l \mathbb{Z}$. Hence, Lemma 2 yields $\left[\sigma_{j}\left(\omega^{m}-\zeta\right), \sigma_{k}\left(\omega^{n}-\zeta\right)\right]=0$ for $j, k \in \mathbb{Z} \backslash l \mathbb{Z}$. Now, let $a \in \mathbb{Z}$ be such that $a \equiv \omega(\bmod l)$. Then $a^{l} \equiv \omega^{l}=\omega\left(\bmod l^{2}\right)$. For $n \in \mathbb{Z} \backslash l \mathbb{Z}$, set $\alpha_{n}^{\prime}=\alpha_{n}\left(a^{l}\right)=a^{l n}-\zeta^{n}$. Then $\alpha_{n}^{\prime} \equiv \omega^{n}-\zeta^{n}\left(\bmod l^{2}\right)$, hence $\left(\bmod \lambda^{l+3}\right)$. On the other hand, $v_{\lambda}\left(\alpha_{n}^{\prime}\right)=v_{\lambda}\left(a^{l n}-(1-\lambda)^{n}\right)=$ $v_{\lambda}\left(a^{l n}-1+n \lambda\right) \leq 1$. It follows, in view of Lemma 3 , that, for all $m, n \in$ $\mathbb{Z} \backslash l \mathbb{Z},\left[\alpha_{m}^{\prime}, \alpha_{n}^{\prime}\right]=\left[\omega^{m}-\zeta^{m}, \omega^{n}-\zeta^{n}\right]$, the latter symbol being equal to $\left[\sigma_{m}\left(\omega^{m}-\zeta\right), \sigma_{n}\left(\omega^{n}-\zeta\right)\right]=0$, by the above. Thus $\left[\alpha_{m}\left(a^{l}\right), \alpha_{n}\left(a^{l}\right)\right]=0$ for all $m, n \in \mathbb{N} \backslash l \mathbb{N}$. Therefore, by [7, Theorem 1$], a^{l} \equiv \pm 1(\bmod l)$, i.e. $\omega^{2} \equiv 1$ $(\bmod l)$, which, as shown in the proof of Theorem 1 , amounts to $\omega^{2}=1$.

COROLlary 3. Under assumption (A), we have:

(1) If $2^{l-1} \not \equiv 1\left(\bmod l^{2}\right)$, then $\omega^{2}=1$.

(2) If $l=2^{n} \pm 1$, for some positive integer $n$, then $\omega^{2}=1$. 
Further, let $l-1=2^{h_{0}} p_{1}^{h_{1}} \ldots p_{m}^{h_{m}}$, where $p_{1}, \ldots, p_{m}$ are distinct odd primes and the $h_{i}$ are positive integers $(0 \leq i \leq m)$.

(3) If $h_{0} \leq 3$ and $p_{i}^{l-1} \not \equiv 1\left(\bmod l^{2}\right)$ for $1 \leq i \leq m$, then $\omega^{2}=1$.

(4) If $h_{0} \leq 3$ and $m=1$, then $\omega^{2}=1$.

(5) If $\sum_{i=1}^{m} h_{i} q\left(p_{i}\right) \not \equiv 1(\bmod l)$, then $\omega^{2}=1$.

(6) If $h_{0} \leq 3$ and, for any proper subset $S \subsetneq\{1, \ldots, m\}, \sum_{s \in S} h_{s} q\left(p_{s}\right) \not \equiv$ $1(\bmod l)$, then $\omega^{2}=1$.

(7) If $h_{0} \leq 3, m=2$ and $h_{i} q\left(p_{i}\right) \not \equiv 1(\bmod l)$ for $i=1,2$, then $\omega^{2}=1$.

Proof. (1) If $f>2$ then, by Theorem $1,2^{l-1} \equiv 1\left(\bmod l^{2}\right)$, contradicting the assumption. Hence $f \leq 2$, i.e. $\omega^{2}=1$.

(2) This is Corollary 1(2).

(3) If $f>2$ then, by Theorem 1 , any $p_{i}$ dividing $f$ should satisfy $p_{i}^{l-1} \equiv 1$ $\left(\bmod l^{2}\right)$, but, in view of the assumption, this cannot occur. Therefore $f \mid 2^{h_{0}}$, and since $h_{0} \leq 3$, we have $f \leq 8$. Hence, by Corollary $2, \omega^{2}=1$.

(4) Since $f$ divides $l-1=2^{h_{0}} p_{1}^{h_{1}}$ and since, by Corollary $1(1)$, the odd prime factor $p_{1}$ of $l-1$ does not divide $f$, we find that $f$ divides $2^{h_{0}}$. Thus $f \leq 2^{h_{0}} \leq 8$, and we conclude as in (3) above.

(5) If $f>2$ then, by Theorem $1,2^{l-1} \equiv 1\left(\bmod l^{2}\right)$, i.e. $q(2) \equiv 0(\bmod l)$. Hence, by [5, Lemma 2] and the assumption, $q(l-1) \equiv \sum_{i=1}^{m} h_{i} q\left(p_{i}\right) \not \equiv 1$ $(\bmod l)$. But this contradicts the congruence $(l-1)^{l-1} \equiv 1+l\left(\bmod l^{2}\right)($ see the proof of Corollary 1$)$, which amounts to $q(l-1) \equiv 1(\bmod l)$.

(6) If $f>2$ then, by Theorem 1 , for any prime $p_{i}$ dividing $f(1 \leq i \leq m)$, $p_{i}^{l-1} \equiv 2^{l-1} \equiv 1\left(\bmod l^{2}\right)$, i.e. $q\left(p_{i}\right) \equiv q(2) \equiv 0(\bmod l)$. Thus, as in the proof of (5) above, $\sum_{p_{i} \nmid f} h_{i} q\left(p_{i}\right) \equiv q(l-1) \equiv 1(\bmod l)$. But, by assumption, no proper subsum of $\sum_{i=1}^{m} h_{i} q\left(p_{i}\right)$ is $\equiv 1(\bmod l)$. Therefore all $p_{i} \nmid f(1 \leq i \leq$ $m$ ), i.e. $f \mid 2^{h_{0}}$, so that $f \leq 2^{h_{0}} \leq 8$, and we conclude as in (3) above.

(7) This is the special case $m=2$ of (6) above.

Corollary 4. Let $l-1=2^{h_{0}} p_{1}^{h_{1}} \ldots p_{m}^{h_{m}}$ be the prime factorization of $l-1$, with $p_{1}, \ldots, p_{m}$ being distinct odd primes and the $h_{i}$ positive integers $(0 \leq i \leq m)$.

The conjecture (WTC) is true for all primes $l$ which satisfy one of the following conditions:

(a) $2^{l-1} \not \equiv 1\left(\bmod l^{2}\right)$.

(b) $l=2^{n} \pm 1$, for some positive integer $n$.

(c) $h_{0} \leq 3$ and $p_{i}^{l-1} \not \equiv 1\left(\bmod l^{2}\right)$ for $1 \leq i \leq m$.

(d) $h_{0} \leq 3$ and $m=1$.

(e) $\sum_{i=1}^{m} h_{i} q\left(p_{i}\right) \not \equiv 1(\bmod l)$.

(f) $h_{0} \leq 3$ and, for any $S \subsetneq\{1, \ldots, m\}, \sum_{s \in S} h_{s} q\left(p_{s}\right) \not \equiv 1(\bmod l)$.

(g) $h_{0} \leq 3, m=2$ and $h_{i} q\left(p_{i}\right) \not \equiv 1(\bmod l)$ for $i=1,2$. 
Acknowledgements. I am grateful for the inspiring friendship, through the years, of Guy Terjanian. In particular, the work on this paper started while I was visiting the University of Toulouse. I would like to thank Guy Terjanian and Bruno Anglès for the gracious hospitality, the stimulating conversations and the congenial atmosphere. I also wish to thank the referee for suggesting substantial simplifications in the paper.

\section{References}

[1] B. Anglès, Units and norm residue symbol, Acta Arith. 98 (2001), 33-51.

[2] E. Artin and J. Tate, Class Field Theory, Benjamin, New York, 1967.

[3] H. Hasse, Bericht über die neueren Untersuchungen und Probleme aus der Theorie der algebraischen Zahlkörper, Teil II: Reziprozitätsgesetz, Jahresber. Deutsch. Math.-Verein., Leipzig, 1930.

[4] - Number Theory, Springer, Berlin, 1980.

[5] C. Helou, Norm residue symbol and cyclotomic units, Acta Arith. 73 (1995), 147-188.

[6] - Power reciprocity for binomial cyclotomic integers, J. Number Theory 71 (1998), $245-256$.

[7] G. Terjanian, Sur la loi de réciprocité des puissances l-èmes, Acta Arith. 54 (1989), $87-125$.

[8] L. C. Washington, Introduction to Cyclotomic Fields, 2nd ed., Springer, New York, 1997.

Pennsylvania State University

25 Yearsley Mill Road

Media, PA 19063, U.S.A.

E-mail: cxh22@psu.edu 